the local Research Ethics Committee and informed consent was obtained from the parents. Venous blood sampling was performed from a peripheral vein. Levels of malondialdehyde (MDA), superoxide dismutase (SOD), reduced glutathione (GSH), and catalase (CAT) were measured before and $24 \mathrm{~h}$ after phototherapy. Statistical analyses were conducted using the SPSS version 20.0. Statistical significance was accepted at $\mathrm{p}<0.05$.

Results The levels of CAT increased after phototherapy in the all groups; however, this increased was not statistically significant $(\mathrm{p}>0.05)$. The levels of GSH in the very preterm group were also found to be decreased significantly after phototherapy. It was observed that levels of MDA were elevated significantly in term neonates as compared to very preterm and late preterm neonates.

Conclusions Phototherapy did increase the oxidative stress in the term, very preterm, and late preterm newborns with jaundice.

\section{PO-0626 WHICH INFANT MANNEQUIN DO YOU PREFER?: USER PREFERENCE AND BAG MASK ABILITY OF DIFFERENT MANNEQUIN MODELS}

G Hawkes, Y Malik, EM Dempsey, CA Ryan. Department of Paediatrics and Child Health, University College Cork, Cork, Ireland

\subsection{6/archdischild-2014-307384.1268}

Background There are a number of infant mannequins available for teaching newborn and infant bag mask ventilation (BMV) skills. These include the NeoNatalie newborn mannequin and the Baby Anne ${ }^{\mathrm{TM}}$ infant mannequin (both Laerdal Medical Foundation, Stavanger, Norway).

Objectives To examine user preference and user ability to perform effective BMV with these mannequin models.

Methods Each participant was randomised to use the Baby Anne $^{\mathrm{TM}}$ infant mannequin (BA) and the NeoNatalie mannequin fully filled with air (NNA), fully filled with water (NNW) and filled with 50\% air and 50\% water (NNAW), each for a 30 second period. Participants were asked to rate the level of fidelity of the mannequins to a "real baby" on a 5-point likert scale in terms of appearance, weight, feel, tone and realism of ventilation.

Results 20 participants completed this study (10 doctors, 10 nurse/midwives).

Fidelity: BA was similar to NNWA in terms of appearance and similar to NNW in terms of weight. However, in terms of touch, muscle tone and realism of ventilation, NNW and NNWA were similar and greatly exceeded BA and NNA.

Breaths: Although all configurations were generally comparable, NNW had the most effective breaths delivered.

Discussion The NNW and NNAW were both shown to have the highest level of fidelity to a "real baby" and had the highest number of effective ventilations delivered. The NNA was disliked in terms of fidelity, and the most difficult to bag due to the difficulty in positioning the airway. This study suggests that NeoNatalie configurations that contain water are the easiest to provide BMV.

\section{PO-0627 OUTCOMES FOR SUBSTANCE MISUSING WOMEN AND THEIR INFANTS 2006-2011: CHANGES OVER A 5 YEAR TIME PERIOD}

K Johnson, M Balain. Neonatology D Floor Martin Wing, Leeds Teaching Hospital NHS Trust, Leeds West Yorkshire, UK

10.1136/archdischild-2014-307384.1269
Background and aims Substance misuse within the UK population continues to be a public health concern. Many of those using illicit drugs are women of childbearing age.

Infants born to such women are at risk of Neonatal Abstinence Syndrome (NAS) and can require a prolonged stay on the neonatal unit. ${ }^{1}$

Understanding of the demographics and outcomes of this vulnerable group of infants and their mothers is vital in order to evolve services to meet needs and improve outcome.

Methods Retrospective review of substance misusing pregnant women and their offspring, 2006-2011.

Basic demographic data and specific outcome measures for the infants was collected over the 5 year period.

Changes over that 5 year time period were explored.

Results 442 women and their infants were included in the study. All infants were admitted for treatment/observation of NAS.

The majority of women were of white British (85.7\%). Opiates were the most commonly misused substances. $18 \%$ of the babies were low birth weight. Breastfed babies were more likely to be discharged within first 7 days of life compared to artificially fed babies $(47.6 \%$ vs $30.6 \%$, OR 1.55 , $95 \%$ CI 0.95 to 2.53).

Conclusion The management of infants with NAS continues to challenge. Breastfeeding leads to reduced intensity of NAS, and should be recommended to shorten length of hospital stay for infants born to substance misusing mothers.

\section{REFERENCES}

1 Dryden $C$, Young D, Hepburn $M$, et al. Methadone use in pregnancy: factors associated with the development of neonatal abstinence syndrome and implications for healthcare resources. BJOG. 2009;116:655-67

\section{P0-0628 REDUCING SERUM BILIRUBIN DETERMINATIONS IN OUTPATIENT NEWBORNS BY SCREENING WITH TRANSCUTANEOUS BILIRUBINOMETRY}

${ }^{1} \mathrm{R}$ Joseph, ${ }^{2} \mathrm{~A}$ Chinnadurai, ${ }^{3}$ WSY Yeo, ${ }^{4}$ PA Nkouibert, ${ }^{5}$ MS George, ${ }^{4}$ ESY Chan, ${ }^{3}$ WE Tang, ${ }^{6} \mathrm{~J}$ Mathews, ${ }^{2} \mathrm{~J}$ Lee. ${ }^{1}$ Neonatology, National University Hospital and Yong Loo Lin School of Medicine, Singapore, Singapore; ${ }^{2}$ Neonatology, National University Hospital, Singapore, Singapore; ${ }^{3}$ Medicine, NHG Polyclinics, Singapore, Singapore; ${ }^{4}$ Epidemiology, Singapore Clinical Research Institute and Duke-NUS Medical School, Singapore, Singapore; ${ }^{5}$ Neonatology, National University Hospiotal, Singapore, Singapore; ${ }^{6}$ Nursing, NHG Polyclinics, Singapore, Singapore

\subsection{6/archdischild-2014-307384.1270}

Background and aim We have previously shown that using Transcutaneous bilirubin (TCB) values that are $90 \%$ of the age specific SBvalue ( $\mathrm{SBPh}$ ) for phototherapy can markedly reduce the need for invasive serum bilirubin (SB) in Day 1-3 inpatient babies. This study aims to determine if the same principle can be applied in older babies in the outpatient setting.

Methods Three to 14 day olds with jaundice at an outpatient care centre in Singapore and needing a SB measurementwere enrolled after obtaining written informed consent. Their TCB level was determined with a commercial bilirubinometer. Correlations and Bias was determined. Using ROC curves, the TCB values that identified the need for an SB were determined.

Results 1072 paired SB and TCB values were obtained from a Chinese (39\%), Malay (35\%), Indian (14\%) and Others (12\%) cohort. Spearman's correlation coefficientsby age groups ranged from 0.882 (2-5 days) to 0.95 ( $>14$ days). The mean bias (SD) was $-8(23.2)$ and the limits of agreement were -54.4 to 38.4 from the Bland Altman analysis. TCB values that were $90 \%$ of the SBPh yielded accuracy and sensitivity rates (\%) of 90 and 
100 in the $<96$ h age group and 96 and 83 in the older group. The efficiency (\% of SB avoided) was 89 and $96 \%$ respectively in the two age groups.

Conclusion TCB measurements that are a predetermined fraction of the SBPh can efficiently reduce SB determinations in the newborn period. The fraction is influenced also by the operational context.

\section{PO-0629 CYCROBILIRUBIN FORMATION CAPACITY AS A NOVEL INDEX IN PHOTOTHERAPY FOR NEONATAL HYPERBILIRUBINEMIA IN A RANDOMISED CONTROLLED STUDY}

${ }^{1} \mathrm{~S}$ Kato, ${ }^{2} \mathrm{H}$ Kakita, ${ }^{3} \mathrm{Y}$ Yamada, ${ }^{4} \mathrm{H}$ Nakashima, ${ }^{5} \mathrm{~T}$ Yamada, ${ }^{6} \mathrm{M}$ Kokubo, ${ }^{7} \mathrm{~K}$ Yokoi, ${ }^{8} \mathrm{~S}$ Fukuda, ${ }^{2} \mathrm{MH}$ Hussein, ${ }^{2} \mathrm{Y}$ Nagaya, ${ }^{2} \mathrm{H}$ Ueda, ${ }^{2} \mathrm{~T}$ Goto, ${ }^{2} \mathrm{~K}$ Ito, ${ }^{2} \mathrm{~T}$ Sugiura, ${ }^{2} \mathrm{~T}$ Ito, ${ }^{2} \mathrm{~K}$ Kato, ${ }^{7} \mathrm{~S}$ Suzuki, ${ }^{2} \mathrm{H}$ Togari. ${ }^{1}$ Pediatrics, Japanese Red Cross Nagoya Daini Hospital, Nagoya, Japan; ${ }^{2}$ Pediatrics, Nagoya-City University, Nagoya, Japan; ${ }^{3}$ Pediatrics, Aichi Medical University, Nagoya, Japan; ${ }^{4}$ Pediatrics, Seirei Hamamatsu General Hospital, Hamamatsu, Japan; ${ }^{5}$ Pediatrics, Tohohashi Municipal Hospital, Toyohashi, Japan; ${ }^{6}$ Pediatrics, Kainan Hospital, Aichi, Japan; ${ }^{7}$ Pediatrics, Nagoya City West Medical Center, Nagoya, Japan; ${ }^{8}$ Pediatrics, Yokkaichi Municipal Hospital, Yokkaichi, Japan

\subsection{6/archdischild-2014-307384.1271}

Aim To examine whether Cycrobilirubin Formation Capacity (CFC) could be clinically relevant in the treatment of neonatal hyperbilirubinemia.

Study design CFC is a sum of irradiances multiplied by the wavelength-specific bilirubin photoisomerization rate, calculated at 40 $\mathrm{nm}$ intervals between the ranges $400-520 \mathrm{~nm}$, measured using a novel spectroradiometer. After evaluating CFC and footprints of light emitting diode (LED) device and fluorescent tube (FT) device, we performed a randomised controlled trial (RCT) to test whether phototherapies provided with different light spectra using the same CFC are equally effective. Thirty-two babies were enrolled without any exclusion criteria. Total serum bilirubin (TSB) levels before and after phototherapies were compared.

Result Seventeen babies were assigned to FT and fifteen to LED group. Birth characteristics were similar. Changes in TSB levels before and after phototherapy in LED and FT group were not different.

Conclusion Under the same CFC evaluated by the spectroradiometer, LED was as effective as FT. This supports the idea that CFC could be relevant in phototherapy for the treatment of hyperbilirubinemia in otherwise healthy newborns.

\section{PO-0630 A RANDOMISED TRIAL OF POSITIONING NEWBORNS ON THE BACK OR RIGHT SIDE FOR UMBILICAL VENOUS CATHETER INSERTION (ISRCTN71265031)}

E Kieran, E Laffan, C O'Donnell. Neonatology, The National Maternity Hospital, Dublin, Ireland

\subsection{6/archdischild-2014-307384.1272}

Background Newborns often have umbilical venous catheters (UVCs) inserted for drug and hypertonic solution administration. Newborns are placed supine for UVC insertion and tip position is confirmed with X-ray. UVCs are considered correctly positioned when the tip is in the inferior vena cava (IVC). Frequently, UVCs cannot be inserted to the desired depth or the catheter is in the portal vanous circulation on X-ray.

Objective To determine whether infants who are placed on the right side for UVC insertion more often have the UVC tip in the IVC compared to infants that are placed on their back.
Methods Infants without major congenital anomalies who were undergoing UVC insertion were randomised to 'BACK' or 'RIGHT SIDE' prior to the procedure. Infants randomised to 'BACK' remained on their back while the UVC was inserted, while those randomised to 'RIGHT SIDE' were turned onto their right side just prior to UVC insertion. The primary outcome for this study was the correct placement of the UVC on X-Ray determined by one Consultant Radiologist who was masked to group assignment.

Results We have enrolled 76 of a planned total of 88 infants since 26/09/2013; we anticipate that recruitment will be complete in June 2014, long before the EAPS 2014.

Conclusions This study will provide valuable information about whether positioning of newborns during UVC insertion affects tip placement.

\section{P0-0631 REVIEW OF THE NEONATAL COMMUNITY OUTREACH TEAM SERVICE FOR INFANTS DISCHARGED ON HOME OXYGEN}

R Krishnamurthy, I Storey, S Shaw, S Moxon, A Singh. Department of Neonatology, Heart of England NHS Trust, Birmingham, UK

\subsection{6/archdischild-2014-307384.1273}

Background The British Thoracic Society has outlined the care pathway for infants with chronic lung disease on home oxygen.

Aim To review the service provided to the infants going home on oxygen, by the Neonatal Community Outreach Team (NCOT) at Heart of England NHS Trust.

Methods NCOT patient notes, Badger database and Parent feedback responses were reviewed to evaluate the care provided to babies on home oxygen.

Results During the 14 month study period, 25 babies on home oxygen were cared for in the community by NCOT. Their median gestation at birth was 27 weeks (23-36 weeks) and median birth weight was 865 grams (520-3180 grams). The median gestation at discharge was 40 weeks (35-64 weeks) with a median oxygen requirement of 0.1 litres/minute (0.02- 0.4 litres/minute). The median number of pre-discharge NCOT contacts was 2 (1-12) and post-discharge contacts 21 (7-36). The median number of post-discharge overnight oxygen saturation studies was 2 (1-7). During the study period, 20 babies came off oxygen with a median post-discharge time of 12 weeks (1-27 weeks). A respiratory syncitial virus vaccination program was successfully completed. The NCOT also facilitated a monthly 'Little Stars' parents meeting and advisory group. The NCOT service had a $100 \%$ satisfaction response on parent survey $(75 \%$ responders).

Conclusion NCOT have provided an excellent service in the community to babies on home oxygen enabling early discharge from neonatal unit whilst appropriately managing oxygen therapy in the community and providing family support and education.

\section{P0-0632 VARIATIONS IN HEALTHCARE WORKER AND PARENTAL PREFERENCES TO SURGICAL MANAGEMENT OF CRITICALLY ILL PRETERM INFANTS}

HS Lam, HM Cheung, CLS Chau, PC Ng. Department of Paediatrics, The Chinese University of Hong Kong, Sha Tin, Hong Kong China

10.1136/archdischild-2014-307384.1274 